\title{
On the issue of additional education for children with special healthcare needs and disabilities in the system of lifelong inclusive education
}

\author{
Elena Medvedeva, Oksana Prikhodko*, Zhanna Zhuravleva, and Anna Pavlova \\ GAOU VO MGPU, ISO \& KR, 119261, Moscow, Russia
}

\begin{abstract}
This article is devoted to one of the most important tasks in the education of children with special healthcare needs (CSHCN) and disabilities to ensure the access to additional learning in various fields: artistic education, natural science, social and pedagogical training, sports and cultural branch, tourist and regional studies, technical studies. The issues of creating special conditions (material, technical, organizational, personnel, curriculum and methodological ones) in institutions of additional education are considered. Two options for organizing additional education for children with special needs and disabilities are presented (in accordance with the criterion for the presence or absence of a conclusion of the Psychological, Medical and Pedagogical Commission - PMPC). Unresolved issues in the field of additional education of children with developmental disorders are described.
\end{abstract}

Currently, the education system for children with special healthcare needs and disabilities faces the most important task to provide the access to lifelong special or inclusive education at all age stages, from the introduction of early assistance to children and their families to the achievement of a high-quality secondary vocational and / or higher education [1].

Inclusive education, upbringing, socialization and social and personal adaptation of children with special healthcare needs and disabilities are the priorities in the Russian Federation. One of the most important tasks of today is to provide the ability to access additional education for such children to enable their interests to be satisfied and developed. Nowadays the issues of additional education for children are the chief concerns of scientists and practitioners, authorities and the general public.

Up to now Russia is one of the few countries that provide state funding for agencies of additional education for children, which is one of the most important focuses of social policy of the Russian Federation, where the integration of education, culture, sports, tourism, social protection, public organizations, etc. takes place. Since 2009, within the framework of the "The Year of Equal Opportunities" curriculum of additional education has become available for children with special healthcare needs and disabilities. Before that, teachers intuitively selected effective methods, techniques and means of teaching such children.

\footnotetext{
${ }^{*}$ Corresponding author: oksanadean@mail.ru
} 
Currently the issues of development and increasing the value of additional education in work with children with special healthcare needs and disabilities are being addressed at the State level, as attested to by the update of the legal framework. The following came into force: the Federal Law "On Education in the Russian Federation", the Concept for the Development of Lifelong Learning, the new "Procedure for the Organization and Implementation of Educational Activities under Additional General Education Curricula". A number of activities of national significance took place: the National Project "Education", the Project "Success of Every Child", the Project "Accessible Further Education for Children" and many others.

Children are given the opportunity to attend children's associations in 6 (six) areas: artistic, natural science, social and pedagogical, physical culture and sports, tourist and regional studies, technical trainings. Physical education, sports and art have dominated among children with special healthcare needs and disabilities.

Children's associations have become inclusive and children with different levels of psychophysical, cognitive, speech and personal development, with different capabilities and abilities are involved in them.

The form of additional education organization is optimal for work with children with disabilities, which involves free choice of creative activity, lack of a rigorous system for evaluating the results and outcomes of studying, the variability of the content and forms of organization of education, convenient system of class attendance, the possibility of conducting individual work with the child and forming the basis for a career choice.

The inclusive capacity of contemporary lifelong learning children with special healthcare needs and disabilities is understood as following: any child with special needs can be included in joint activities with peers and teachers while the individual abilities of children are revealed; every child is aware of its significance and value a long as normally developing children are taught empathy, support and mutual assistance. The possibility of successful socialization and social and cultural development in an atmosphere of equality, cooperation, productive communication is carried out.

On the one hand the advantage of additional education for students with disabilities is seen due to the free choice of the activity type that children like. On the other hand its strength lies in the variability of the forms and contents of the educational process, its flexibility and mobility. In some cases, additional education helps children to compensate their school failures with the help of achievements in additional education.

Children with special healthcare needs and disabilities can receive additional education in the kindergarten, school, in the organizations of additional education, leisure centers, clubs, libraries, etc. Most of the children study in general developmental curricula. In the absence of medical contraindications, they will be able to master the pre-professional curriculum, which allows identifying talented children among students with special healthcare needs and disabilities, for whom this type of activity (primarily in the field of art, physical education and sports) may subsequently become the only opportunity for their professional self-determination.

A number of studies have been devoted to the issues of additional education for children with special healthcare needs and disabilities (S. Alekhina, O. Butorina, O. Vitvar, A. Volkov, V. Golovanov, E. Dikaeva, I. Drobakhina, J. Zhuravleva, O. Zabolotskikh, I. Markevich, E. Medvedeva, A. Pavlova, I. Solovieva and others). Their analysis of the studies has showed that this problem is presented to a greater extent in pedagogical works, rather than psychological ones. Despite the development of a system of training, education and development of children with disabilities, some issues are not well understood. This concerns the peculiarities of including such children in joint activities with normally developing peers in the context of further education. It is required to address the issues of development and testing of the content, methods and techniques of teaching, forms of 
personal development of children. It is necessary to search and identify the most effective psychological and pedagogical technologies etc, in the field of additional education [2].

It should be stressed that the priority in the additional education of children with disabilities is not the mastery of specific knowledge, but the ability to apply this knowledge successfully in social and life situations. First of all, this is the personal outcomes of children with disabilities. These include adaptation of the child to new conditions, satisfaction from their activities, increase in creative activity, display of initiative and curiosity, the establishment of productive collaboration with peers and teachers, tolerance towards others, development of vital, social competencies, such as autonomy, responsibility, the ability to be interested in others and taking part in their lives and the ability to set goals and achieve them.

In accordance with the Federal Law on Education, today each organization must meet the special needs and create special conditions, necessary for children with disabilities to master any curriculum.

Traditionally there are two typical approaches of organizing additional education (in accordance with the criterion for the presence or absence of a conclusion of the Psychological, Medical and Pedagogical Commission - PMPC). According to the first option, a child does not have a conclusion of PMPC or an individual rehabilitation program when admitted to institutions of additional education for children with special healthcare needs. In this case, it is advisable to obtain all the relevant information about the disorders from the parents or legal representatives of the child. Therefore this option should be characterized as of spontaneous integration, not inclusion.

The second scenario deals with the presence of the PMPC conclusion or an individual rehabilitation program with recommendations made on creating special educational conditions. This includes material and technical conditions, which involve creating an accessible architectural environment, organization of a workplace with special equipment for children with disabilities. Unfortunately, not all institutions of additional education have created a barrier-free environment.

Apart from the legal support of the educational process and creating appropriate financial and economic conditions organizational conditions also imply provision of real psychological and pedagogical support for all participants in the educational process. Furthermore that includes distance learning of handicapped children, creating a correctional and developmental environment, access to the information on the website of the specialized educational institution for children their parents (the websites of many regional model centers do not correspond the needs of children with disabilities and their families, which makes it difficult for parents to find the necessary information), creating an atmosphere of emotional comfort.

We will address in more detail to the psychological and pedagogical support in the absence of which the cognitive and personal development of such children will be arduous. Psychological and pedagogical support is a holistic, interconnected system, including variable correctional technologies and increasing the effectiveness of social adaptation of children with developmental problems. It helps to increase the level of their upbringing, personal development and successful socialization. Synergy of teachers and tutors of additional education, psychologists, parents and the administration of educational organization is vital for the successful assistance to the child at all age stages [3].

The main role in organizing the assistance belongs to a psychologist, however not all organizations of additional education has one on a full-time position. Unfortunately, there is a lack of other specialists such as speech pathologists, speech therapists, social educators, etc. in organizations of additional education. Beyond determining the level of development of each child a psychologist specifies the methods and directions of correctional work, coordinates the activities of all participants of educational process. The assistance is carried 
out in the framework of measures organized the psychologist and during the classes of teachers of additional education. All the positive results achieved by children are recorded by parents and the immediate social environment. The main principles of this process are priority interests of the assisted, sustainability of assist, its focus, system and flexibility, a complex approach, consistency at different levels of education, network collaboration and recommendations of specialists involved in the assist [1].

Personnel conditions are considered realized if psychological and pedagogical consultations function steadily in the institution of additional education. Amongst other things teachers of additional education need to complete advanced training courses (at least 72 hours) or to master a professional retraining program on the issues of education, raising children with disabilities. The analysis of psychological and pedagogical research (S. Alekhina, T. Belyakova, V. Golovanov, S. Seryakova and others), as well as our personal practical experience has showed insufficient awareness of teachers of additional education about the specific features of development children with special healthcare needs and disabilities, on methods and technologies for corrective work with children of various nosologies, on the development and adaptation of an additional general curriculum. Understanding the specifics of violations, knowledge of the actual and potential abilities of children will help them to be accepted and feel success in a pleasant and attractive activity and to increase the professional competence of additional education teachers [4]. The teacher needs special knowledge, psychological and methodological support, and constant professional interaction with colleagues who have such experience. The staff of the institution of additional education should include a psychologist, a speech pathologist, a speech therapist and a tutor. There should be collaboration of all the specialists involved in the assistance, an additional education teacher and parents. It results in the mutual enrichment and complementarity of each participant, which helps to optimize the pedagogical process in the context of inclusive additional education.

The curriculum and methodological conditions include the development of adapted additional curricula for children with disabilities and educational and methodological equipment of the educational process. Currently, in most additional general educational curricula, there is no correctional aspect: correctional goals and objectives are not highlighted, the principles of special psychology and pedagogy are not taken into account, methods, work directions, means, measures, technologies that need to be used when working with children with disabilities are not described. It is important to take into account the specific laws of development and special educational needs for different types of dysontogenesis, because the tactics of the approach to each child, the choice and creation of special conditions for children of various categories of developmental disorders depend on this. The specific features of the correctional orientation when working with such children are not always taken into account in the curriculum and scientific and methodological support of the educational process while planning to provide an opportunity for children with disabilities to join children clubs [4].

One of the unresolved issues in the field of additional education is the lack of consensus for medical recommendations with indications / contraindications for classes and exercise regimen. Parents of children with disabilities, teachers of additional education could use this information when choosing a direction, curriculum when entering an institution of additional education, taking into account the interest of the child and his real capabilities, drawing up a specific educational and developmental route for each student.

Institutions of additional education at the regional level have gained some experience working with children with special healthcare needs and children with disabilities. This is evidenced by the statistics of the effectiveness of measures (annual increase in winners, laureates, diploma winners, champions of various levels of contests, festivals, sports and athletics competitions, competitions, etc.). 


\section{References}

1. O.G. Prikhodko and others, The activities of specialists are accompanied by the training of disabled children in the educational space (GBOU VPO MGPU, Moscow, 2014)

2. E.A. Medvedeva, Kazan pedagogical journal, 3 (122), 132-138 (2017)

3. E.A. Medvedeva, A.S. Pavlova, The formation of personality of primary schoolchildren with mental retardation in the conditions of additional education (Pero, Moscow, 2018)

4. J.I. Zhuravleva, A.S. Pavlova, Problems of modern pedagogical education, 60-3, 126129 (2018) 